\title{
Experimental investigation into localized instabilities of mixed Rayleigh-Bénard-Poiseuille convection
}

\author{
EMERIC GRANDJEAN AND PETER A. MONKEWITZ† \\ Laboratoire de Mécanique des Fluides, Ecole Polytechnique Fédérale de Lausanne (EPFL), \\ CH-1015, Lausanne, Switzerland
}

(Received 15 January 2009; revised 31 July 2009; accepted 1 August 2009)

The stability of the Rayleigh-Bénard-Poiseuille flow in a channel with large transverse aspect ratio (ratio of width to vertical channel height) is studied experimentally. The onset of thermal convection in the form of 'transverse rolls' (rolls with axes perpendicular to the Poiseuille flow direction) is determined in the Reynolds-Rayleigh number plane for two different working fluids: water and mineral oil with Prandtl numbers of approximately 6.5 and 450, respectively. By analysing experimental realizations of the system impulse response it is demonstrated that the observed onset of transverse rolls corresponds to their transition from convective to absolute instability. Finally, the system response to localized patches of supercriticality (in practice local 'hot spots') is observed and compared with analytical and numerical results of Martinand, Carrière \& Monkewitz (J. Fluid Mech., vol. 502, 2004, p. 175 and vol. 551, 2006, p. 275). The experimentally observed two-dimensional saturated global modes associated with these patches appear to be of the 'steep' variety, analogous to the one-dimensional steep nonlinear modes of Pier, Huerre \& Chomaz (Physica D, vol. 148, 2001, p. 49).

Key words: absolute/convective, instability, Bénard

\section{Introduction}

The Rayleigh-Bénard-Poiseuille (RBP) system is an open Rayleigh-Bénard (RB) cell heated from below with a unidirectional through-flow at a sufficiently low Reynolds number to maintain a laminar parabolic velocity profile. It is noted that the addition of through-flow to the RB cell is in many respects analogous to adding axial through-flow to the closely related Taylor-Couette system as discussed by Chomaz (2005). A schematic of the present RBP system is shown in figure 1 together with the coordinate system used in the following. With and without throughflow, this system develops a thermo-convective instability as soon as pressure and buoyancy forces associated with temperature-induced density perturbations are large enough to overcome viscous forces and to set fluid in motion before heat diffusion can significantly reduce the driving density perturbation. In the classical laterally unconstrained RB system without through-flow, this motion takes the form of convection rolls of arbitrary orientation because of the rotation symmetry of the base state around any vertical axis. With unidirectional through-flow this symmetry is broken, and one must distinguish between two principal roll directions: longitudinal 


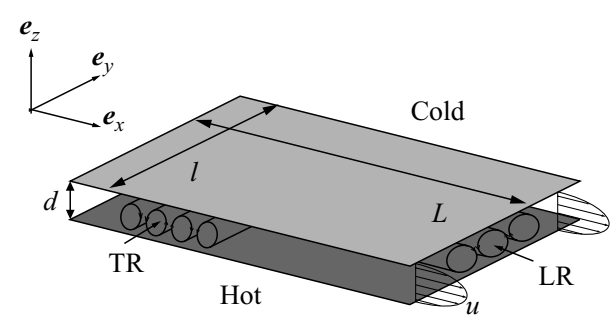

FIGURE 1. Schematic of RBP system between two horizontal plates at $z= \pm d / 2(d=4 \mathrm{~mm})$ with Poiseuille flow in the $\boldsymbol{e}_{x}$ direction. Also shown are longitudinal rolls (LR) with axes $\| \boldsymbol{e}_{x}$ and transverse rolls (TR) with axes $\| \boldsymbol{e}_{y}$.

rolls with axes parallel to the flow direction and transverse rolls perpendicular to the flow direction, henceforth denoted by LRs and TRs, respectively, and included schematically in figure 1 . Since the streamwise direction $x$ is a homogeneous direction of the RBP system with LRs present the stability boundary for LRs is the same in the RB and the RBP system. Only the streamlines associated with LRs change from closed loops to helices. The TRs on the other hand form a chain of counter-rotating roll pairs pushed along by the mean flow. These structures are two-dimensional (except near the sidewalls) since the transverse $y$ direction is a homogeneous direction of the RBP system with TRs. For a thorough review of the characteristics of the RBP system, see Nicolas (2002).

The stability characteristics of the RBP system are determined by the following three main parameters and, to a certain degree, also by the path on which specific parameter values are reached:

(i) The Rayleigh number

$$
R a=\frac{g \beta\left(T_{\text {hot }}-T_{\text {cold }}\right) d^{3}}{\nu \alpha},
$$

with $g$ the gravitational acceleration, $\beta$ the volumetric thermal expansion coefficient, $T_{\text {hot }}$ and $T_{\text {cold }}$ the internal surface temperatures of the lower (hot) and upper (cold) channel walls, respectively, $d$ is the vertical channel height, $v$ the kinematic viscosity and $\alpha$ the thermal diffusivity of the fluid evaluated at the mean temperature $\left(T_{\text {hot }}+\right.$ $\left.T_{\text {cold }}\right) / 2$.

(ii) The Reynolds number $R e$ is based on the maximum Poiseuille flow velocity $U_{\max }$ calculated from the volumetric flow rate under the assumption of steady, fully developed flow,

$$
R e=\frac{U_{\max } d}{v} .
$$

(iii) The Prandtl number $P r$, finally, is the ratio of viscous to thermal diffusivities,

$$
\operatorname{Pr}=\frac{v}{\alpha} \text {. }
$$

The above three parameters completely determine the state of an RBP system with a Newtonian working fluid which is of infinite horizontal extent, has perfectly conducting channel walls and has a small enough temperature difference $\left(T_{\text {hot }}-T_{\text {cold }}\right)$ to justify the approximation due to Boussinesq (1903), where all fluid properties are evaluated at $\left(T_{h o t}+T_{\text {cold }}\right) / 2$ except for the density in the buoyancy term. Such an idealized system has been the object of several theoretical studies. While the absolute 


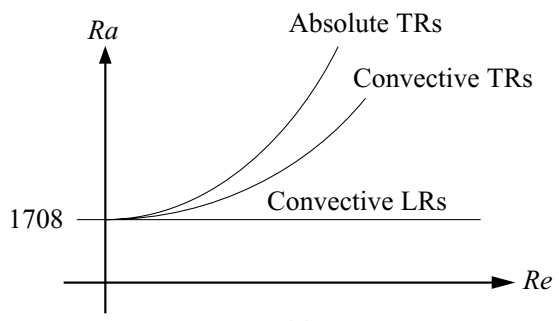

(a)

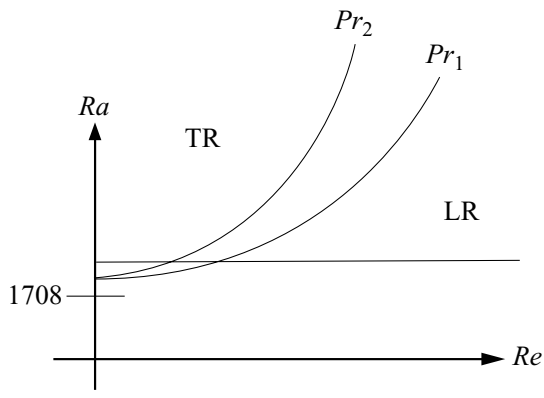

(b)

FIGURE 2. (a) Stability diagram of the idealized RBP system of infinite horizontal extent with perfectly conducting horizontal boundaries. The solid lines are the stability boundaries of LRs and of TRs and the convective-absolute transition for TRs (bottom to top). (b) Schematic of the effect of finite transverse aspect ratio according to Luijkx et al. (1981) for two Prandtl numbers $\left(\operatorname{Pr}_{2}>\operatorname{Pr}_{1}\right)$. No scales are indicated, since they depend on $\operatorname{Pr}$ and $A R_{\perp}$.

nature of the TR instability at high enough $R a$ has been accepted since the pioneering work of Müller (1990), the same question for LRs has only later been clarified by Carrière \& Monkewitz (1999). The resulting stability diagram is shown schematically in figure 2(a): LRs become unstable at the same $R a_{c}^{L R}=1708$ as the pure RB system, independent of $R e$, and they remain, always convectively unstable, as long as $R e>0$. The stability boundary $R a_{c}^{T R}(R e)$ for TRs, on the other hand, is roughly a quadratic function of $R e$ for small $R e$ with a curvature that increases strongly with $P r$. As $R a$ is increased beyond $R a_{c}^{T R}(R e)$, the TRs become increasingly convectively unstable until a convective-absolute transition is reached at $R a_{a b s}^{T R}(R e)$ which is again approximately a quadratic function of $R e$ in the range of small $R e$ considered here.

As soon as a more realistic system is considered, additional control parameters come into play. The first one is the transverse aspect ratio $A R_{\perp}$ (channel width $/ d$ ). As $A R_{\perp}$ is reduced, the stability boundary for LRs is pushed to higher Rayleigh numbers faster than the one for TRs. While these shifts are imperceptible at $A R_{\perp}=42.5$ used in this study, they become experimentally relevant for $A R_{\perp}$ values of less than, say, 4 when TRs become the dominant mode over a sizeable Reynolds number interval as shown schematically in figure $2(b)$. Details on the effects of the Prandtl number and aspect ratio can be found in Luijkx, Platten \& Legros (1981), Kato \& Fujimura (2000) and Nicolas, Luijkx \& Platten (2000).

The comparison between theory and experiment is further complicated by the fact that most theoretical studies of the RBP system use the Boussinesq approximation, whereas in the present study temperature-induced viscosity variations can reach $50 \%$ at the highest Reynolds numbers, where a large temperature difference is needed to reach the high $R a_{c}^{T R}$ of TRs. Large viscosity variations are known to promote different convection modes due to the breaking of vertical symmetry, but in the present study non-Boussinesq effects had at most a quantitative effect on the results. This is supported by the study of Stengel, Oliver \& Booker (1982) who investigated the effect of viscosity variations on pure $\mathrm{RB}$ convection and found that a $50 \%$ variation induces an increase of $R a_{c}$ of the order of only a few per cent. No comparable study exists for the RBP system except the one by Sameen \& Govindarajan (2007) at high Reynolds number at which the system is rather a channel flow modified by heating. For the present case of (very) low Reynolds number we therefore surmise that the effect is similar to the one found in the RB system. Furthermore, in our experiment 
which required optical access to the test section, the horizontal walls are relatively far from being perfectly heat conducting because they were made of glass and not the more standard copper as in most other experiments. In our set-up the ratio between the thermal diffusivities of fluid and glass $\alpha_{\text {fluid }} / \alpha_{\text {wall }}$ was 0.12 for oil and 0.21 for water. According to Hurle, Jakeman \& Pike (1967) this reduces $R a_{c}$ in pure RB convection by $4 \%$ and $7 \%$, respectively, and also slightly reduces the wavenumber. The limit of large $\alpha_{\text {fluid }} / \alpha_{\text {wall }}$, on the other hand, has been investigated by Busse $\&$ Riahi (1980) and Chapman \& Proctor (1980). For the present small $\alpha_{\text {fluid }} / \alpha_{\text {wall }}$ and small Re we again assume a behaviour of the RBP system similar to the one reported by Hurle et al. (1967).

Because of the various difficulties, only few experimental studies of the RBP system exist, and even fewer experiments have been aimed at elucidating the properties of TRs. In the latter category is the work of Trainoff (1997) who worked with a long and narrow spiral-shaped channel of $A R_{\perp}=2.2$ and streamwise aspect ratio $A R_{\|}=59$. As this $A R_{\perp}$ is sufficiently low to make TRs the dominant mode over a sizeable range of $R e$, Trainoff was able to study the response of TRs to noise and their transition to absolute instability without being bothered by LRs.

In the present paper we study RBP flow experimentally in a channel of large transverse aspect ratio. The first aim is to determine the convective-absolute transition $R a_{a b s}^{T R}$ of TRs and to compare with the linear theoretical results of Müller (1990) and Carrière \& Monkewitz (1999). To avoid any ambiguity, this transition is determined by two independent methods: as bifurcation to self-sustained TRs and from the experimental impulse response. Due to the large $A R_{\perp}$, the principal challenge in these experiments has been to avoid triggering convectively unstable longitudinal rolls before the TRs of interest are observed. Pictorially, this amounts to 'tiptoeing' very quietly under the most unstable LRs in order to get at the TRs. The second purpose of the present study is the experimental characterization of the system response to a localized supercritical island, a 'hot spot', in a subcritical 'sea'. More specifically, we are looking at global modes associated with a localized region of absolute instability in order to assess the asymptotic and numerical studies of Martinand, Carrière \& Monkewitz $(2004,2006)$ who concluded that such global modes consisted of a packet of essentially transverse rolls. Experimentally only the final saturated global mode was accessible without an absolute amplitude calibration, and hence the comparison with the above-mentioned studies remains qualitative. However, the experiments raise interesting questions on the connections between the one-dimensional nonlinear global modes constructed by Pier, Huerre \& Chomaz (2001), in particular their 'steep' mode, also called 'elephant' mode in Pier \& Huerre (2001), and the two-dimensional global modes observed in our RBP set-up.

\section{Experimental set-up}

In the first subsection, the RBP channel is described in detail together with the temperature and flow rate control systems, the technique to produce localized heating of the bottom plate and the optical set-up. The second subsection is devoted to the experimental procedures and diagnostics used to obtain the results of $\S 3$. For more details on the experimental set-up the reader is referred to Grandjean (2008).

\subsection{RBP facility}

The actual test section consists of two $5.5 \pm 0.2 \mathrm{~mm}$ transparent floated borosilicate windows of $170 \mathrm{~mm}$ width and $220 \mathrm{~mm}$ length (in the flow direction $\boldsymbol{e}_{x}$ ), mounted in 


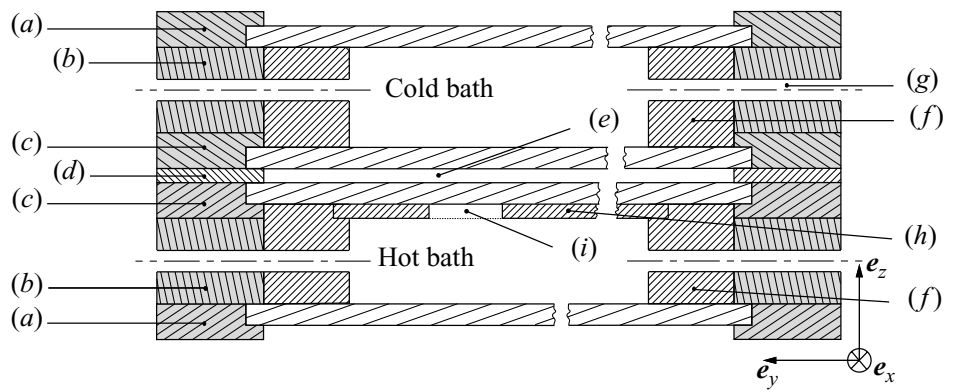

FIGURE 3. Cross-section of RBP facility (vertical spanwise plane $\boldsymbol{e}_{y}-\boldsymbol{e}_{z}$ normal to the flow direction): $(a),(b)$ Frames and transparent covers of the heating and cooling baths; $(c)$ brass frames and windows of the test section; $(d) 4 \mathrm{~mm}$ spacer; $(e)$ test section; $(g)$ inlets/outlets for the temperature-controlled cooling and heating circuits; $(f)$ added Plexiglas frames within the heating and cooling baths to provide 'soft' thermal boundary conditions on the periphery of the RBP channel; $(h)$ removable additional thermal resistance between hot bath and test section used to produce localized 'hot spots' at the cut-out (i).

brass frames separated by a $4 \mathrm{~mm}$ Plexiglas (PMMA) spacer. The windows have a nominal thermal conductivity of $1.2 \mathrm{~W}(\mathrm{mK})^{-1}$ and were fitted into the brass frames with sufficiently thick silicone joints to allow for differential thermal expansion. The waviness of the windows, their elastic deflection due to pressure differences and mounting errors in the brass frames resulted in a total error of the gap height of $\leqslant 1 \%$.

The inlet of the channel is located $200 \mathrm{~mm}$ upstream of the actual heated test section in a Plexiglas supply tank of $320 \times 320 \mathrm{~mm}^{2}$ cross-section, and the edges of the inlet are rounded to form a bell mouth. The supply tank has a free surface, and its length of $600 \mathrm{~mm}$ is subdivided by a vertical porous layer $100 \mathrm{~mm}$ thick consisting of $4 \mathrm{~mm}$ plastic spheres into a quiet section with the channel inlet and a section in which the return flow is introduced. Thermal convection in the quiet inlet section is eliminated by using symmetric heating and cooling of the test section, which keeps the working fluid in the thermally insulated inlet tank at the mean laboratory temperature. Immediately downstream of the test section the working fluid is collected through a spanwise slit at the bottom of the channel followed by a vertical channel of the same width filled with a $140 \mathrm{~mm}$ high layer of small spheres to a level $80 \mathrm{~mm}$ below the test section. This second porous layer ensures that the transverse pressure gradient, i.e. the spanwise variation of flow velocity, is negligible all the way to the end of the test section.

The working fluid is circulated by a pulsation-free gear pump (Micropump, model GB-P35 with integrated bypass) operated at a high internal flow rate of around $10^{5} \mathrm{~mm}^{3} \mathrm{~s}^{-1}$ to minimize flow unsteadiness and vibrations in the system. Its output flow rate is adjusted between 0 and about $500 \mathrm{~mm}^{3} \mathrm{~s}^{-1}$ with a continuous electrovalve, computer controlled by a self-made capillary flowmeter. Two different working fluids are used in order to test the strong $P r$ effects: white mineral oil with a nominal $\operatorname{Pr}$ of 450 and distilled water with $P r \approx 6.5$ at laboratory temperature.

As shown in figure 3 the test section is sandwiched between a heating and a cooling bath each consisting of a PVC frame and an outside glass cover to maintain optical access to the test section. These baths are incorporated into water circuits thermally regulated by two Lauda RK8KP thermostats with double-action pumps to achieve large flow rates with minimum unsteadiness at small pressure drops. 
To minimize the influence of the lateral boundaries on the onset of undesirable longitudinal rolls, 'soft' lateral boundary conditions (i.e. a gradual reduction of $R a$ to subcritical conditions in a narrow strip adjacent to the sidewalls of the test section) were added. For this purpose, frames were inserted into the heating and cooling baths to make the effective heating and cooling areas narrower than the test section by $20 \mathrm{~mm}$ on each side.

For the experiments with non-uniform heating of the bottom plate, two arrangements were used. To produce an experimental approximation of a hot Dirac impulse in space and time on the bottom surface of the test section, a dot of black paint of about $5 \mathrm{~mm}$ diameter and 10-15 $\mu \mathrm{m}$ thickness was sprayed on the inside bottom wall of the test section $50 \mathrm{~mm}$ from its upstream edge (the start of the heated part of the channel). This dot could be heated during a short time (15-30s proved optimal for the present experiments) by aiming a continuous argon-ion laser beam at it from the top with up to $1.5 \mathrm{~W}$ output power. Steady non-uniform heating of the bottom of the test section, on the other hand, has been produced with a spatially non-uniform thermal resistance placed against the underside of the glass bottom of the test section. Specifically, a $4 \mathrm{~mm}$ thick Plexiglas plate ( $h$ in figure 3 ) is added in the hot bath and held against the bottom window of the test section by grooves in the frame ( $f$ in figure 3 ). To obtain the desired shape and location of a hot spot, appropriate cut-outs were provided in this Plexiglas plate, where the water of the hot bath could enter into direct contact with the bottom glass window. Motivated by the theoretical studies of Martinand et al. (2004, 2006), two different shapes were used: a circular hole of $40 \mathrm{~mm}$ diameter and a 'racetrack oval' of $15 \times 50 \mathrm{~mm}$ inclined $45^{\circ}$ with respect to $\boldsymbol{e}_{x}$. The centre of both cut-outs was located on the centreline $65 \mathrm{~mm}$ from the leading edge of the test section. In order to produce a hot spot of the same shape as the cut-outs, it proved necessary to cover the cut-outs by a fine mosquito screen in order to eliminate local high velocities of the heating water within the cut-outs.

To observe such a sensitive system, only non-invasive diagnostics can be used. Furthermore, techniques requiring seeding particles such as laser Doppler anemometry and particle image velocimetry (PIV) are excluded because at the very low flow velocities in the present experiments (down to $0.08 \mathrm{~mm} \mathrm{~s}^{-1}$ ) even a slight variation of the fluid density leads to particle deposits on the top or the bottom wall of the test section and to subsequent pinning of the thermal convection structures. Therefore, a modified Z-type schlieren system (see Settles 2001) is used. A short arc $(0.6 \times 2.2 \mathrm{~mm})$ mercury vapour bulb (Osram $200 \mathrm{~W}$ HBO $200 \mathrm{w} / 4$ ) is placed at the focal point of a parabolic mirror of $300 \mathrm{~mm}$ diameter in order to produce a large parallel beam aimed through the channel. The perturbed output beam is then focused on a diaphragm to create the schlieren image which is recorded by a standard 8 bit CCD camera (model A301 by Basler with up to 80 non-interlaced frames per second of $654 \times 494$ pixels). With the mineral oil as working fluid, which has a high temperature sensitivity of the refractive index, the axisymmetric diaphragm of the camera lens itself was used to produce the schlieren image. With the smaller refractive index sensitivity of water, the errors due to imperfections of the schlieren system and of the RBP facility became comparable. Therefore it was necessary to have a more precise control over the positioning of the cutoff device. Since a pinhole provided insufficient light for the camera, a razor blade, which has the disadvantage of being sensitive only to variations normal to the blade edge, had to be used as cutoff device for the water experiments. 
While in principle this system integrates refractive index variations over the test section plus the heating and cooling baths, a sufficiently long exposure time allows to observe only the variations associated with the thermo-convective instabilities in the test section, as the small index of refraction variations in the heating and cooling baths where flow velocities are high occur on a much shorter time scale than those associated with the instabilities of interest.

\subsection{Experimental procedures}

In the following, the physical properties of the working fluid are always given at its mean temperature $T_{\text {mean }}$ which was chosen equal to the average laboratory temperature.

\subsubsection{Determination of the Rayleigh number $R a$}

Since the flow channel requires full optical access for the observation of roll pattern, the top and the bottom of the channel are made of borosilicate glass which has a relatively low thermal conductivity. Therefore, the temperature difference $T_{\text {hot }}-T_{\text {cold }}$ applied to the fluid layer has to be computed from the applied bath temperature $T_{\text {hot }}^{\text {bath }}$ and $T_{\text {cold }}^{\text {bath }}$. Assuming a pure conductive state, $T_{\text {hot }}-T_{\text {cold }}$ follows simply from the continuity of conductive heat flux using the following thermal conductivities for Borofloat glass, oil and water: $\kappa_{\text {glass }}=1.08 \mathrm{~W}\left(\mathrm{~m}^{\circ} \mathrm{C}\right)^{-1}$ at $25^{\circ} \mathrm{C}$, $\kappa_{\text {oil }}=-7.39 \times 10^{-5} \mathrm{~T}+0.1376$ and $\kappa_{\text {water }}=-8.6 \times 10^{-6} T^{2}+0.0021 T+0.56$ with $T$ specified in degree Celsius. It is noted that this procedure provides the correct $T_{\text {hot }}-T_{\text {cold }}$ up to the onset of thermal convection, independent of the Reynolds number, except near the upstream edge of the heated/cooled section. Considering that the oncoming fluid has the mean temperature between the hot and cold baths, the development length from this upstream test-section edge that is necessary to establish a linear temperature profile is estimated as $(d / 2) \operatorname{Re} P r$ which has been $\leqslant 7 d$, i.e. $\leqslant 28 \mathrm{~mm}$, in all the experiments reported here, except at the highest velocities of figure 9.

Even though we are mainly interested in the critical conditions for the onset of thermal convection and the region close to onset where the difference between $R a_{\text {cond }}$ based on conduction alone and the true $R a_{\text {cond }+ \text { conv }}$ is small, it is useful to estimate the evolution of this difference. This is possible for $R e=0$ with the experimental Nusselt numbers of Silveston (1958). The resulting relation between $R a_{\text {cond }}$ and $R a_{\text {cond }}+$ conv is shown in figure 4. Note however that the ratio of fluid and wall conductivities of Silveston were not the same as in the present experiments. For $R e>0$ no such data are available, but the influence of the through-flow on the Nusselt number is small as long as the vertical velocity associated with the convection rolls is large compared to their translation velocity. This ratio can be estimated as $\operatorname{Re}^{-1} \operatorname{Pr}^{-1 / 2}\left(R a-R a_{c}\right)^{1 / 2}$ which is considerably larger than 10 in all the experiments presented here.

\subsubsection{Determination of the Reynolds number and assessment of flow quality}

The Reynolds number is based on the 'maximum' velocity $U_{\max }$ of the Poiseuille flow. To deduce it from the flow rate $Q$ measured with the capillary flowmeter and the dimensions of the channel, the ratio between the maximum and mean velocities needs to be determined. According to White (1974), the corresponding ratio of Reynolds numbers for the present transverse aspect ratio of 42.5 is

$$
R e=\frac{U_{\text {max }} d}{v}=\frac{1.52 U_{\text {mean }} d}{v} .
$$




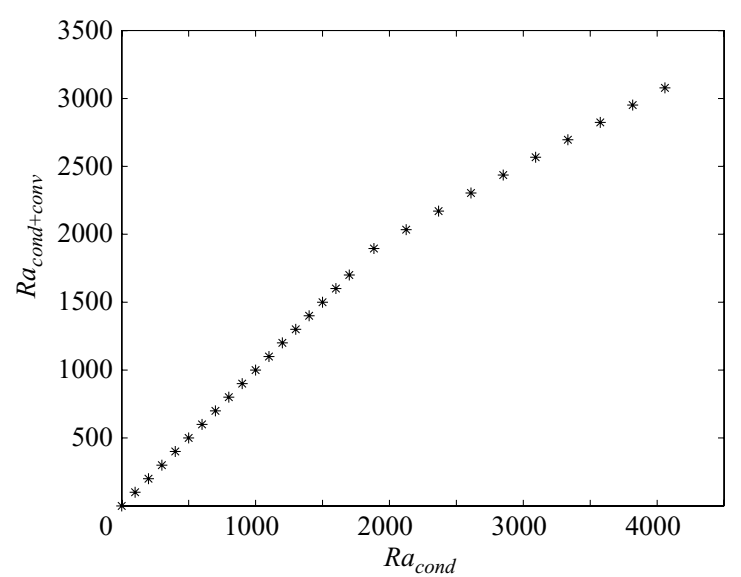

FIGURE 4. Effect of thermal convection on $R a$ in the pure RB case with water: $R a_{\text {cond }}+$ conv obtained from the experimental Nusselt data of Silveston (1958) (vertical axis) versus $R a_{\text {cond }}$ computed for the same heat flux with heat conduction alone. The slight discontinuity at $R a_{c}=1708$ is due to experimental errors of the Nusselt number.

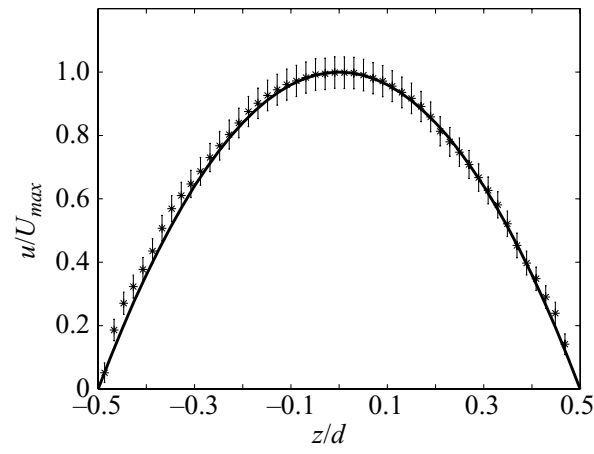

(a)

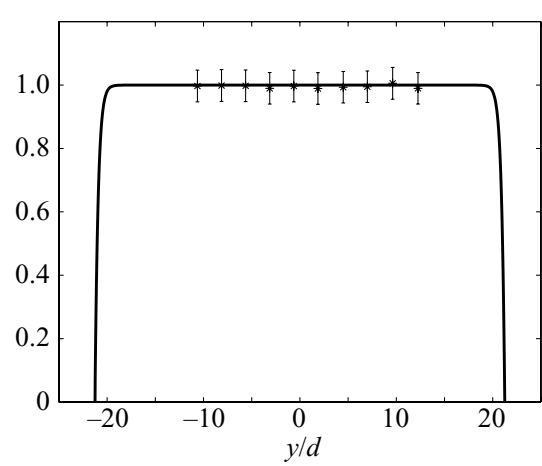

(b)

FIgURE 5. Comparison of theoretical velocity profiles (solid lines) and PIV measurements ( $\star$ ), normalized with the theoretical maximum velocity $U_{\max }$. (a) Vertical profile at mid-span $y=0$; (b) spanwise profile at mid-height $z=0$.

Result (2.1) presupposes a parabolic profile with a constant maximum velocity across the channel, except near the sidewalls. This has been verified with PIV at $R a=0$, i.e. without thermo-convective structures, using seeding particles of average diameter of $5 \mu \mathrm{m}$. A continuous-wave laser sheet was aimed into the channel (without heating/cooling baths) in such a way as to obtain a light sheet in the working section that was parallel to $\boldsymbol{e}_{x}$ and inclined $45^{\circ}$ with respect to $\boldsymbol{e}_{z}$. (The laser was aimed at the channel at a computed external angle in order to compensate for the refraction through the upper window.) The camera was positioned symmetrically to view the laser sheet at right angles. A mapping function was used to correct for the optical distortion of the images by the upper window. Data processing was performed with the open source MatPIV code (Sveen 2004) using multi-pass mode and interrogation windows of $16 \times 16$ pixels. One vertical velocity profile $u(y=0, z)$ at mid-span is shown in figure $5(a)$, while the spanwise distribution of maximum velocity $u(y, z=0)$ is shown in figure $5(b)$. These measurements were non-dimensionalized with the 
maximum velocity of the theoretical profile obtained from the flow rate and hence demonstrate their excellent agreement with the theoretical Poiseuille flow.

\subsubsection{Quantification of the schlieren image}

To obtain at least semi-quantitative results from a schlieren image, the measured intensity must be converted to refractive index variations $(\propto$ temperature variations). However, both a fully experimental calibration of the schlieren system and the complete theoretical determination of the transfer function proved prohibitively complex. We simulated a two-dimensional RB cell with finite elements and the schlieren set-up with geometrical optics to try to relate different image contrasts to the amplitude of convection rolls. Unfortunately it proved impossible to lower the error of the diagnostics below $30 \%$ because of too many sources of error such as pixel saturation, impossibility to simulate the complex camera lens and uncertainty about the critical alignment of the diaphragm or knife edge. A further uncertainty is introduced into the simulation by a CCD fill factor of only $75 \%$, meaning that $25 \%$ is blind surface around every light-sensitive pixel which is needed for connectivity.

Therefore, the data treatment was limited to producing relative amplitudes of the rolls within each experimental run. Due to the extreme sensitivity of the schlieren image to minute changes of the alignment and the light source, amplitudes of different runs could not be compared. For the study of TRs in the uniformly heated case, the roll amplitude was determined from a two-dimensional discrete Fourier transform of $480 \times 480$ pixels corresponding to an area of $9.6 \times 9.6 \mathrm{~mm}^{2}$. Hence, the highest resolved wavenumber is $(\pi / d) /(12 \pi)$, where $(\pi / d)$ is the approximate roll wavenumber. As the schlieren signatures of rising and falling fluid are different, the lowest Fourier peak corresponds to the true roll wavenumber. The roll amplitude is then estimated by integrating over $5 \times 5$ spectral points centred on the lowest Fourier peak.

To obtain the spatial amplitude distribution of a saturated global mode associated with the two hot spots described above, a different image treatment is required. In a first step, an image of the test section at subcritical conditions is recorded during start-up of every experimental run and is then subtracted from every image in order to eliminate as much as possible the mosquito screen covering the cut-out as well as stationary optical defects. Any remaining high-wavenumber noise is then removed by a two-dimensional finite impulse response (FIR) band-pass filter centred on the fundamental roll wavenumber. Finally, the envelope of global modes is determined as the absolute value of the complex image consisting of the filtered experimental image as real part and its Hilbert transform as imaginary part (Oppenheim \& Schafer 1989). As the global modes associated with absolutely unstable hot spots consist essentially of TRs, the FIR Hilbert transform was only applied in the flow direction $\boldsymbol{e}_{x}$.

\subsubsection{Characterization of varying surface temperatures}

The temperature distribution of the lower surface of the test section produced by the cut-outs of the extra thermal resistance ( $i$ in figure 3 ) was investigated with thermochromic liquid crystal (TLC) sheets. Using a proper hue/temperature calibration provided a resolution of $\pm 0.5^{\circ} \mathrm{C}$ and allowed in particular to determine the actual shape of the absolute instability boundary $R a_{a b s}^{T R}(x, y, R e)$ of transverse rolls as a function of $R e$ for both cut-out shapes. As an example, temperature profiles for the racetrack oval at $R e=0$ and $R a=1690$ are shown in figure 6 . 

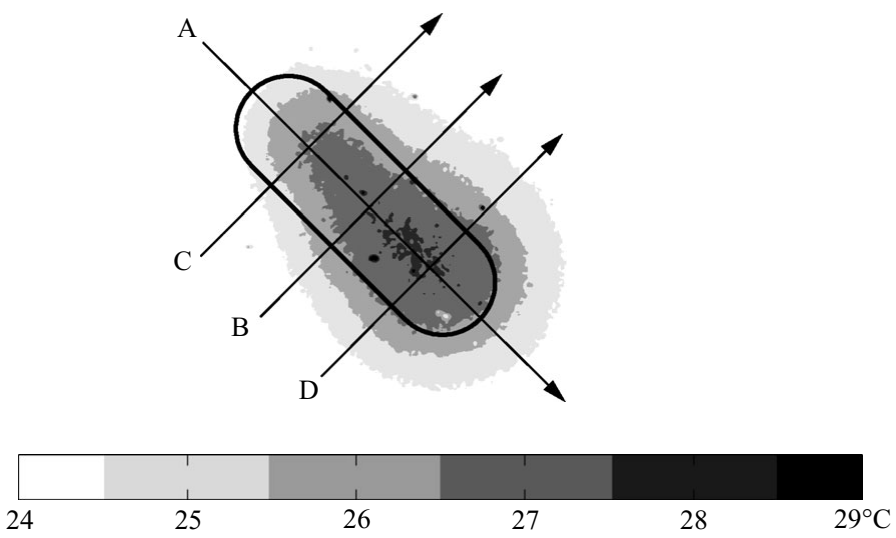

(a) $R e=0 R a=1690$

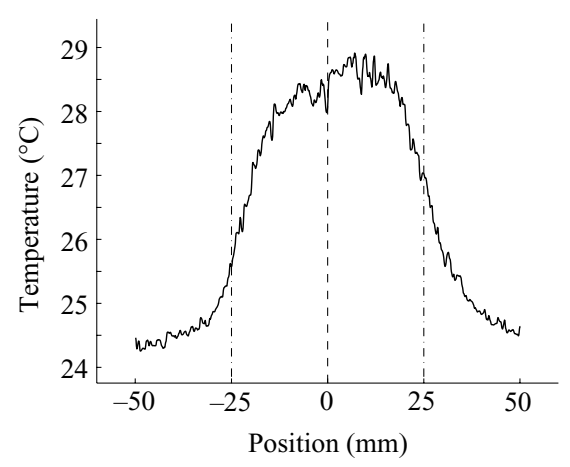

(b) Profile along A

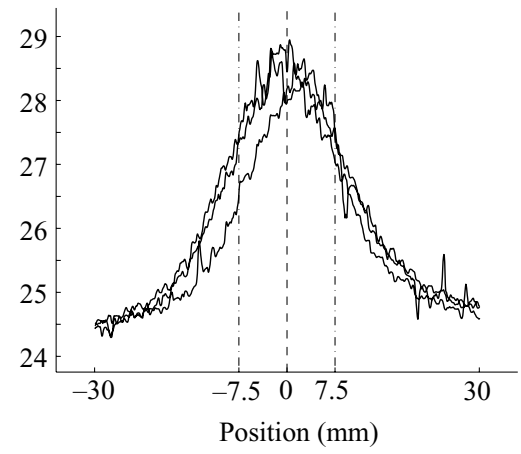

(c) Profiles along B, C and D

FIGURE 6. Measured temperature profiles produced by the $15 \mathrm{~mm} \times 50 \mathrm{~mm}$ racetrack oval cut-out using TLC at $R a=1690$ and $R e=0$. (a) Temperature map computed pixel-by-pixel from TLC calibration curve; $(b),(c)$ temperature profiles in the different sections defined in $(a)$.

\section{Results and conclusions}

In the first two subsections the experimental stability characteristics of the RBP system at low $R e$ with uniform heating and cooling of the bottom and top walls are presented. The aim is to determine the convective-absolute transition for TRs. This programme is hampered by the fact that LRs in a large RBP cell become convectively unstable at a lower $R a$ than the TRs, independent of $R e$. It is nevertheless possible to avoid any measurable perturbation of the base state within the finite length of the test section up to the point at which TRs become self-excited because LRs never become absolutely unstable for $R e>0$ (Carrière \& Monkewitz 1999). Therefore LRs can be avoided by sufficiently reducing or eliminating all sources of their excitation. This has been a major difficulty of this study, as our system had to be made excessively quiet (see also Ouazzani et al. 1995). This requirement can only be relaxed if the transverse aspect ratio is drastically reduced to the point of making TRs more unstable than LRs at low $R e$, as in the experiments of Trainoff (1997) with $A R_{\perp}=$ 2.2 .

After successfully avoiding LRs, there still remains the question of whether TRs appear in the experiment only at and beyond the convective-absolute transition, 
as assumed in $\S 3.1$, or also in convectively unstable situations. The latter is highly unlikely, since the excitation of convectively unstable TRs requires highly spanwise coherent, i.e. two-dimensional, perturbations which are not expected to be contained in the uncontrolled 'experimental noise' of the present set-up. This interpretation of the results of $\S 3.1$ are verified with impulse response experiments in $\S 3.2$. In the third subsection, thermo-convective patterns are investigated which are due to localized heating of the bottom plate, i.e. due to a local patch of super-criticality (including an area of absolute instability) in an otherwise stable channel. The resulting global modes are in most cases clearly two-dimensional analogues of the onedimensional steep nonlinear modes of Pier et al. (2001). This is further confirmed by comparison of experimental saturation amplitudes with available numerical results.

\subsection{Appearance of transverse rolls at the convective-absolute boundary}

As said above, it is assumed that in the experiment TRs first appear spontaneously after crossing the boundary between convective and absolute instability. To determine the critical Rayleigh number $R a_{a b s}^{T R}$ for the convective-absolute transition of TRs as a function of $R e$, runs at about ten different fixed Reynolds number were carried out with each fluid. Each run started from a completely settled system at the laboratory temperature and proceeded through step increases of the temperature difference between the cooling and the heating bath, typically to $R a \approx 3000-6000$ (corresponding to a maximum temperature difference of approximately $25^{\circ} \mathrm{C}$ for oil and $10^{\circ} \mathrm{C}$ for water). After each slow temperature increase of around $1^{\circ} \mathrm{C}$ the system was left to settle for $40 \mathrm{~min}$, during which time temperature and the thermo-convective pattern could reach equilibrium. This equilibration time corresponds to 12 and 20 thermal diffusion times $\left(d^{2} / \alpha\right)$ for mineral oil and water, respectively. Only then images of the roll pattern were taken and the next temperature increase was started. To further minimize the undesirable appearance of LRs, the temperature steps were chosen such as to avoid the settling period in a region of the stabililty diagram (figure 2) where LRs are unstable and TRs still stable whenever this was possible, i.e. when the width of this region corresponded to a temperature difference of no more than $1^{\circ} \mathrm{C}$. Each experimental run lasted about $12 \mathrm{~h}$ and was performed during the night to minimize environmental perturbations, vibrations from nearby vehicle traffic in particular.

To extract the $R a$ of the convective-absolute transition (at constant $R e$ ) for TR we use Landau's theory (see Drazin \& Reid 2004) for a standard supercritical Hopf bifurcation. It holds that beyond the bifurcation, the saturation amplitude of the fundamental squared is proportional to the super-criticality parameter, in this case proportional to the difference between the Rayleigh number and $R a_{a b s}^{T R}$ of the convective-absolute transition:

$$
\left|\mathrm{A}_{0}\right|_{\text {sat }}^{2} \propto\left(R a-R a_{a b s}^{T R}\right) .
$$

As usual, for every fixed $R e$ the bifurcation is located by recording TRs at several supercritical Rayleigh numbers (typically around 10), determining their fundamental amplitude by Fourier analysis (see $\S 2.2$ ) and least squares fitting these amplitudes with (3.1). The justification for this approach comes from both experiment and theory. On the one hand, the data correlate well with (3.1) (see Grandjean 2008), and the theoretical analysis of Kato \& Fujimura (2000) has shown that the bifurcation to TRs is supercritical for $\operatorname{Pr}=7$ and $A R_{\perp}$ down to unity. 


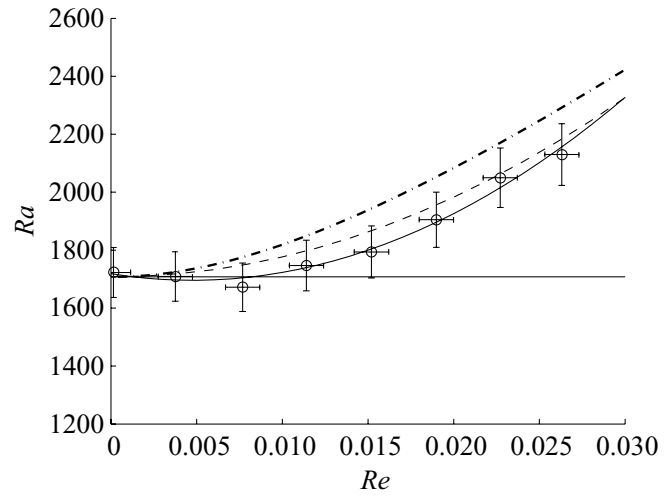

(a) Oil, $\operatorname{Pr} \approx 450$

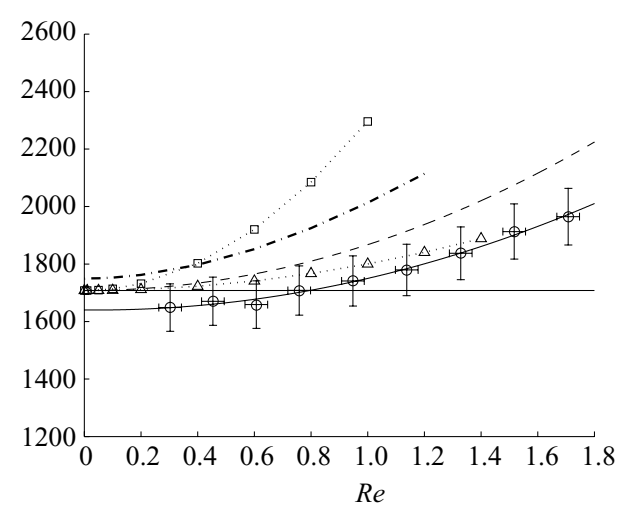

(b) Water, $\operatorname{Pr} \approx 6.5$

FIGURE 7. Stability boundaries in the $R a-R e$ plane for $(a)$ oil and $(b)$ water. The continuous lines are the least square quadratic fits (3.2) of the present experimental onset of TRs $(O)$. The dash-dotted curve is the convective-absolute transition of TRs evaluated numerically by Carrière \& Monkewitz (1999), while the dashed line is from the DNS of Müller, Lücke \& Kamps (1992). The dotted lines marked by ' $\triangle$ ' and ' $\square$ ' are measurements by Trainoff (1997) of $R a_{c}^{T R}$ and $R a_{a b s}^{T R}$, respectively, for water. The horizontal continuous line provides the reference $R a=1708$.

The results for oil and water are shown in Figures $7(a)$ and $7(b)$, respectively. The circles with error bars are the present experimental measurements corresponding to the convective-absolute transition in figure 2. The solid lines are least squares quadratic fits of the experimental $R a_{a b s}^{T R}$ given by

$$
\begin{aligned}
R a_{a b s}^{T R}(\text { oil }) & =9.296 \times 10^{5} R e^{2}-9.897 \times 10^{3} R e+1.723 \times 10^{3}, \\
R a_{a b s}^{T R}(\text { water }) & =1.199 \times 10^{2} R e^{2}-1.010 \times 10^{1} R e+1.640 \times 10^{3} .
\end{aligned}
$$

Also included in figure 7 is the two-dimensional direct numerical simulation (DNS) of Müller, Lücke \& Kamps (1992) (dashed line) and the convective-absolute transition computed by Carrière \& Monkewitz (1999) (dash-dotted line). Finally, the experimental convective ' $\triangle$ ' and absolute ' $\square$ ' instability boundaries of Trainoff (1997) are included in the right-hand graph to show the strong stabilizing effect of his very small transverse aspect ratio $\left(A R_{\perp}=2.2\right)$, but it is noted that according to Kato \& Fujimura (2000), his $R a_{a b s}^{T R}(R e=0)$ should be around 2380. For the present data, one notices that the experimental convective-absolute transition curve lies below the theoretical and numerical curves. This difference is attributed to the finite heat diffusivity of the glass windows as opposed to the infinite diffusivity assumed in the theoretical and numerical studies. For our set-up, the ratios of heat diffusivities $\alpha_{\text {fluid }} / \alpha_{\text {wall }}$ are 0.12 and 0.21 for oil and water, respectively. According to Hurle et al. (1967) these ratios reduce the 'standard' value of $R a_{c}(R e=0)=1708$ by about 70 and 120 for oil and water, respectively, which is fully consistent with the error bars in figure 7.

It is interesting to note that while the effect of the finite heat diffusivity of the walls on $R a_{a b s}^{T R}$ is relatively weak, the phase speed of the TRs is strongly affected. While the Reynolds numbers in oil were too low to obtain reliable measurements, the phase speed of TRs in water could be measured at a sufficiently supercritical $R a=4000$ with an estimated error of $\pm 6 \%$ in the Reynolds number range $0.4 \leqslant R e \leqslant 1.6$. In 
this range, the result is well approximated by the ad hoc correlation

$$
c_{\text {phase }}(\mathrm{TR} @ R a=4000) / U_{\text {mean }} \approx 0.6235 \exp (-4.076 R e)+0.9466 .
$$

The extrapolated phase speed at $R e=0$ compares reasonably well with the values of around 1.4 reported by Ouazzani, Caltagirone, Meyer \& Mojtabi (1989) and Nicolas et al. (2000). The physical reason for this strong effect of $R e$ is the small thermal diffusion velocity in the wall $\left(\alpha_{\text {wall }} / 2 d\right)$ based on approximately one roll wavelength $(\approx 2 d)$ which, for water at $R a=4000$, is smaller than the flow velocity beyond $R e \approx 0.013$ and therefore acts as a 'brake' on the propagation of TRs.

\subsection{Convective-absolute transition from the impulse response}

In the previous subsection it has been argued that in our experiment TRs first appear at the convective-absolute instability boundary and not at the traditional (convective) instability boundary. To support this, the impulse response of the present system has been studied experimentally. It permits the unambiguous determination of the convective or absolute nature of the instability by measuring the propagation direction of the upstream edge of the wave packet as shown for the RBP system by Carrière \& Monkewitz (1999).

To measure the impulse response of the system, the system was stabilized in the desired supercritical state. Then a temporary fast flow rate during typically 5-10 s was used to sweep the thermo-convective rolls out of the test section. Then, the flow rate was rapidly reduced to the desired $R e$, and the system was left to settle for 1-2 min (depending on $\mathrm{Pr}$ ) in order to obtain a stationary and convection-free supercritical system. At this time, the black target of $5 \mathrm{~mm}$ diameter on the bottom surface of the channel was heated by a laser pulse of $0.8-1.5 \mathrm{~W}$ output power during $15-30 \mathrm{~s}$. Two examples of the response to this experimental approximation of a Dirac heat input, as recorded by the schlieren system, are shown in figures $8(a)$ and $8(c)$, where $(a)$ clearly represents an absolutely unstable situation, as the impulse response has spread upstream of the black target, and $(c)$ a convectively unstable case.

In principle, the 'edge' of the impulse response is defined as the contour separating amplified from damped perturbations. In practice, the edge visible on a schlieren image is not well defined. Since the stationary black dot did not allow the use of the Hilbert transform to determine the envelope of the wave packet, we have chosen to determine the position of upstream and downstream edges 'by hand': it is defined as the outermost coherent contrast emerging from the noise of the schlieren image. As the wave packet continuously adds new approximately circular rolls at its circumference, this technique has a tendency to produce jumps in the edge positions. However, the envelope of the visual edge positions appears to be a good approximation of the true wave-packet edge. To obtain the edge velocities, the edge positions are fitted linearly. In cases in which the system is contaminated by TRs and/or LRs originating from perturbations other than the laser impulse, the fits are limited to early times as in figure $8(b)$.

The measurements of wave-packet edge velocities as a function of the Reynolds number $R e$ for a fixed $R a \approx 2000$ are summarized in figures $9(a)$ and $9(b)$ for oil and water, respectively. The transition from absolute to convective instability is clearly identified as the $R e$ at which the upstream edge velocity (stars on the graphs) becomes positive, i.e. at which the entire impulse response starts moving downstream. Considering all the uncertainties, this $R e_{a b s}^{T R}$ from the impulse response compares well with the convective-absolute transition in figure 7 obtained from the Hopf bifurcation 


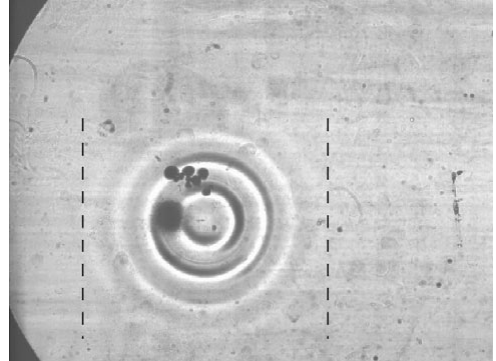

(a)

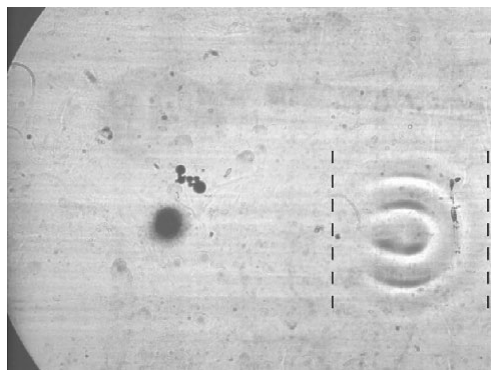

(c)

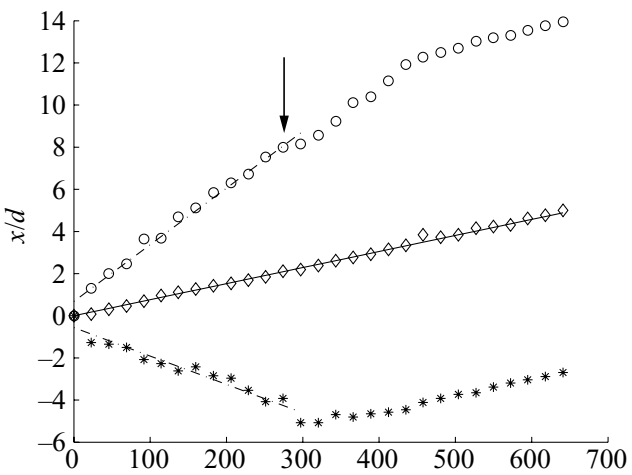

(b)

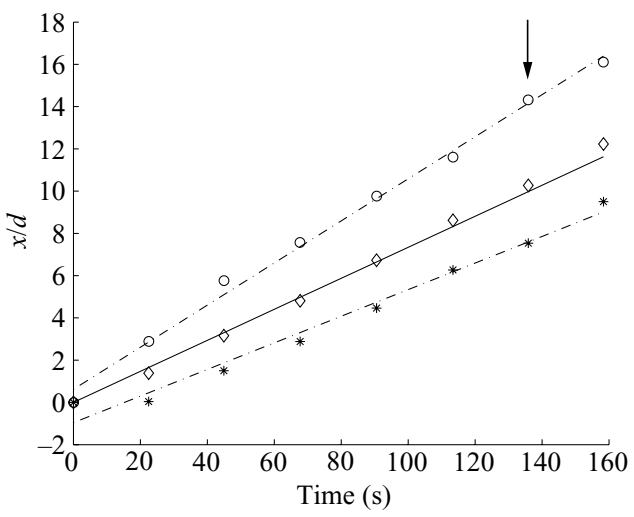

(d)

FiguRE 8. $(a)$ and $(b)$ Impulse response in oil $(P r=450)$ at $R e=0.0064$ and $R a=1970 .(a)$ Raw schlieren image with vertical broken lines marking the upstream (left) and downstream (right) edges of the wave packet. $(b)$ Trajectories of upstream edge $(*)$, downstream edge $(O)$ and centre $(\diamond)$ of wave packet; _- trajectory at mean Poiseuille flow velocity; $-\cdot-\cdot-$, least squares fits of edge trajectories; $\downarrow$, time of schlieren image $(a) .(c)$ and $(d)$ Analogous to $(a)$ and $(b)$ but for oil at $R e=0.064$ and $R a=2060$.

of TRs in the uniformly heated system and indicated by the vertical arrows in figure 9 .

\subsection{The response to steady localized heating}

In the final part of this study, the response of the RBP system to a single 'island' of localized supercritical heating in the middle of a subcritical 'sea' is explored. The hot islands are created by a hole in an additional thermal resistance on the heating bath side of the lower channel wall, as described in $\S 2.1$. The main goal of these experiments is to observe the 'saturated' thermo-convective global modes produced by two different supercritical islands previously studied analytically and numerically.

The experimental procedure is the same as the one used for the detection of the transverse roll onset. A step-by-step $R a$ increase is imposed on the system while $R e$ is kept constant, and the system is left to settle the entire night after the final value of $R a_{\max }$ (maximum Rayleigh number inside the hot island) is reached. Only then a schlieren image of the final stationary saturated global mode is recorded. Thereby $R a_{\max }$ is chosen to be the largest value which allows $R a_{\infty}$ (Rayleigh number far from the localized heating area) to stay in the subcritical range with the given thermal 


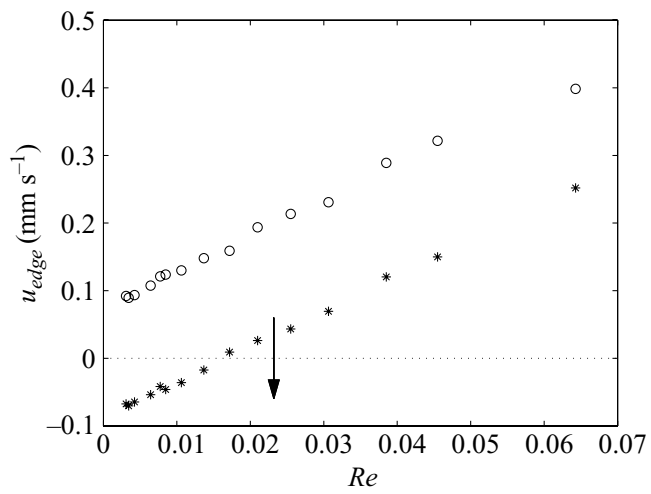

(a) Oil $(\operatorname{Pr} \approx 450)$

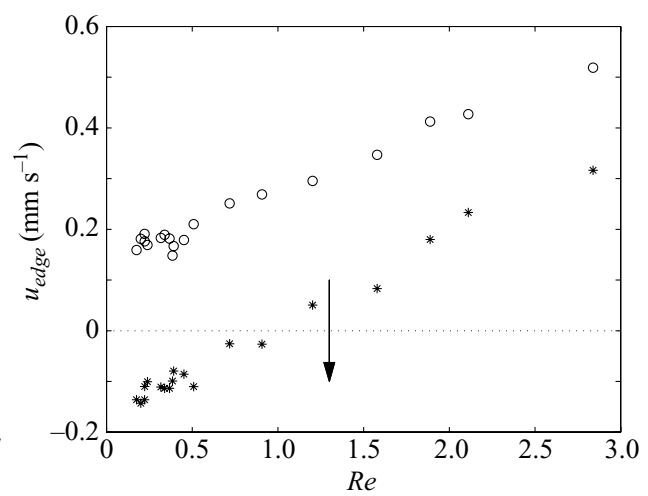

(b) Water $(\operatorname{Pr} \approx 6.5)$

FigURE 9. Edge velocities of the impulse response versus $R e$ for $R a \approx 2000: *$, upstream edge; $O$, downstream edge; $\downarrow$, convective-absolute transition for $R a \approx 2000$ from figure 7 .

resistance. Since $R a_{c}$ for TRs, which dominate in global modes at $R e>0$, is an increasing function of $R e$, a large value for $R a_{\max }$ is necessary to compare global modes at a fixed $R a$ and different $R e$.

The amplitude distribution of the saturated localized convection pattern is then determined by the Hilbert transform technique, as described in $\S 2.2$, but it has not been possible to obtain consistent amplitudes between different experimental runs. This is because the schlieren system has to be realigned after every experimental run, and even minute changes of the cutoff-device position produces large differences of contrast. Hence, the amplitudes are without scale in the following figure 10, which shows the system response to localized heating in the form of an oblique racetrack oval and of a circle for oil as working fluid. No results for water are shown because of the weak schlieren contrast. To facilitate interpretation, both the roll pattern and a three dimensional plot of the envelope are given in each case. The dark grey colouring of the envelopes indicates where the underlying base state is locally absolutely unstable as determined from the surface temperature measured with liquid crystals in a separate experiment (see $\S 2.2$ ) and the measured transition curve fitted by (3.2). The light grey colouring, on the other hand, lumps local convective instability or stability which are not distinguished, as this would require a full stability analysis with the actual boundary conditions.

Looking first at the two images in figures $10(a)$ and $10(d)$ for $R e=0$, the global mode is seen to have the same shape as the hot spot and to be centred on it, i.e. on its locally absolutely unstable region, as expected from the symmetries of the no-flow base state, i.e. rotation symmetry for the circular hot spot and two reflection symmetries for the racetrack oval (neglecting the distant boundaries of the test section). Note that the rolls in figure $10(a)$ are aligned with the short side of the racetrack oval, analogous to the roll pattern in a box of low aspect ratio.

As soon as the flow is turned on, these symmetries are broken as seen in figures $10(b), 10(c), 10(e)$ and $10(f)$, and the rolls forming the global modes are aligned approximately normal to the flow or in other words are TRs independent of the shape of the hot spot. This is entirely consistent with the notion that a localized global mode is generally associated with an area of local absolute instability and that only TRs become absolutely unstable in the RBP system. Note that figure 10(c) corresponds 


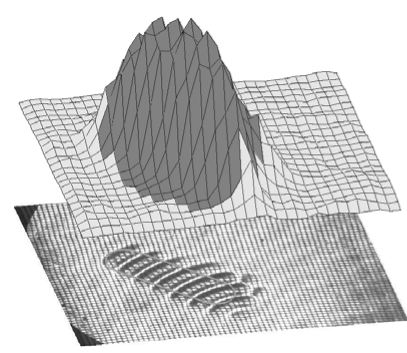

(a) $R e=0.000, R a_{\max }=2682$

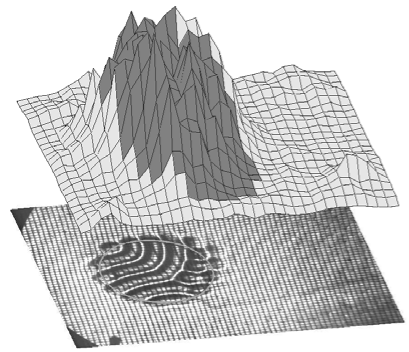

(d) $R e=0.000, R a_{\max }=2615$

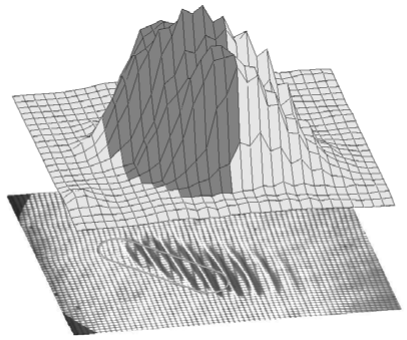

(b) $R e=0.015, R a_{\max }=2635$

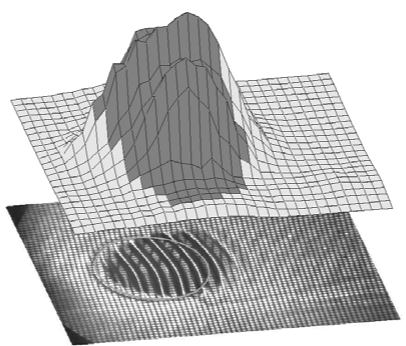

(e) $R e=0.009, R a_{\max }=2542$

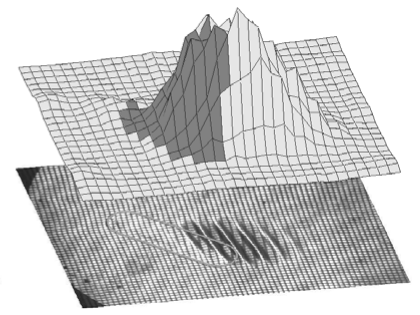

(c) $R e=0.021, R a_{\max }=2656$

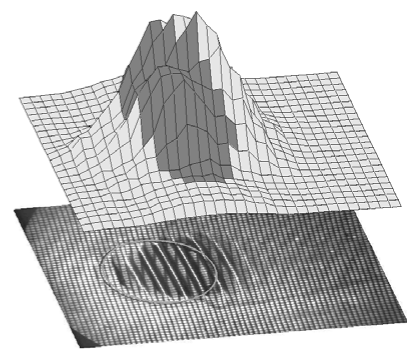

(f) $R e=0.018, R a_{\max }=2666$

FIGURE 10. Envelope of global modes for $P r=450, R a \approx 2600$ and different $R e$ with absolutely unstable area in dark grey. The original schlieren images with the outline of the cut-out in the thermal resistance are shown below each amplitude distribution. $(a)-(c)$ Hot spot in the form of an oblique racetrack oval; $(d)-(f)$ circular hot spot. The Poiseuille flow direction is from left to right in all images. Note that the vertical scale does not change within the sequences $(a)-(c)$ and $(d)-(f)$ but is different in both sequences.
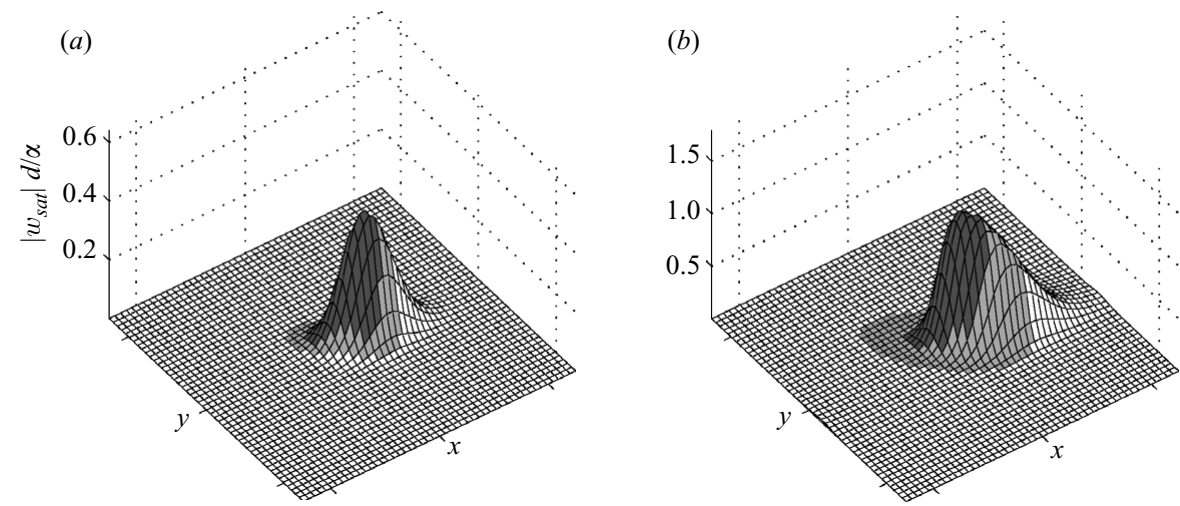

FIGURE 11. Normalized vertical component $w$ of the perturbation velocity of the nonlinear saturated modes computed by Martinand et al. (2006) for a swept elliptical temperature spot. (a) $\operatorname{Pr}=7, \operatorname{Re}=0.38$ and $R a_{\max }=1800$ while $R a_{\infty}=1500 ;(b) \operatorname{Pr}=7, \operatorname{Re}=0.85$ and $R a_{\max }=2000$ while $R a_{\infty}=1500$. Dark, medium and light grey correspond, respectively, to absolute instability, convective instability and stability of TRs.

to an $R e$ close to the stability boundary of the global mode, while the highest $R e$ for the circular hot spot in figure $10(f)$ is well within the unstable region.

Figure 10 is clearly in qualitative agreement with the theoretical analysis and the numerical simulations of global modes by Martinand et al. (2004, 2006). For an easier comparison, two of these computations for a swept elliptical hot spot similar to the experimental racetrack oval are reproduced as figure 11. Despite that 


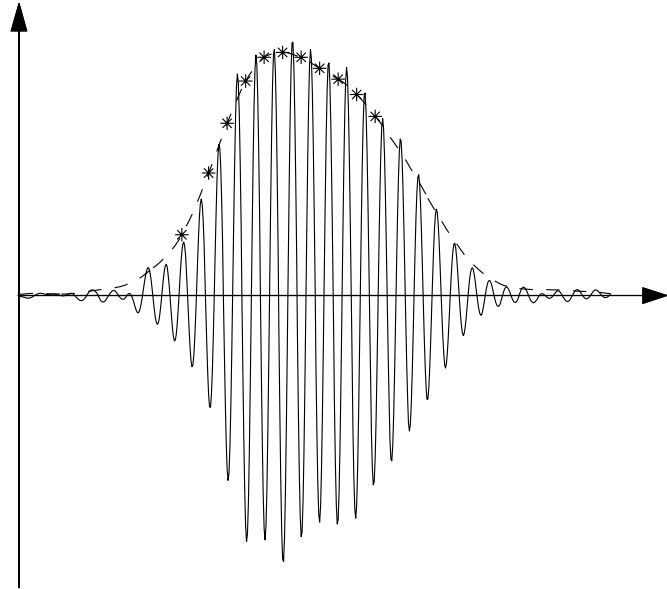

FIGURE 12. Streamwise amplitude profile corresponding to a streamwise slice of figure $10(b)$ : — , schlieren signal band-pass filtered at the fundamental roll wavenumber; - - -, wave-packet envelope with stars indicating local absolute instability.

the computations were made for water as a working fluid, the similarities with figures $10(b)$ and $10(c)$ are striking. Of particular interest is the question whether the saturated global modes in the RBP system are the two-dimensional equivalent of 'steep' or 'soft' one-dimensional nonlinear global modes first analysed by Pier et al. (2001). Even though the global modes of figure 10 appear to be 'attached' to the most upstream portion of the absolute instability boundary, analogous to the steep one-dimensional modes, it is difficult to fully answer the question based on the three-dimensional plots. Therefore, the case of figure $10(b)$ for $\operatorname{Pr}=450, \operatorname{Re}=0.015$ and $R a_{\max }=2635$ is analysed in more detail. First, to increase the signal-to-noise ratio, the original image has been band-pass filtered in the streamwise direction at the roll wavenumber (note that this produces an average of the schlieren signatures of rising and falling fluid which are different). Then a streamwise slice approximately through the centre of the wave packet was selected, and the envelope of this slice containing about 10 rolls was computed by Hilbert transform. The result, after a final spline smoothing, is displayed in figure 12 without amplitude scale, since no calibration of the schlieren system was possible. This streamwise slice through the saturated global mode of figure $10(b)$ clearly shows a difference between the steeper front and the flatter back. Hence, for $R e>0$ our two-dimensional global modes have all the essential characteristics of the one-dimensional steep global modes constructed by Pier et al. (2001):

(a) The steep front of the saturated global mode is seen to be 'attached' to the most upstream part of the convective-absolute boundary.

(b) The global mode consists of structures which become locally absolutely unstable at its front; i.e. it consists of TRs.

(c) The flatter back of the global mode extends well into the downstream convective and stable regions.

Note that the length of the wave packet does not figure in the above list, as it depends on the extent of the absolutely unstable and the downstream convectively unstable regions. Hence, all the evidence points in the direction of the saturated global modes of figure 10 being two-dimensional equivalents of 'steep' one-dimensional global modes. A definitive conclusion will however have to await the extension of nonlinear 
global mode theory to two dimensions which will in particular have to establish the conditions for the existence of the upstream nonlinear front in the presence of transverse inhomogeneity.

The authors would like to thank Dr Navid Borhani for his valuable help in setting up this experiment and gratefully acknowledge the financial support of the Swiss National Science Foundation under grant no. 200020-208177.

\section{REFERENCES}

BoussinesQ, J. 1903 Théorie analytique de la chaleur, mise en harmonie avec la thermodynamique et la théorie mécanique de la lumière, Tome II: Refroidissement et échauffement par rayonnement. Conductibilité. Courant de convection. Gauthier-Villars.

Busse, F. H. \& Riahi, N. 1980 Nonlinear convection in a layer with nearly insulating boundaries. J. Fluid Mech. 96, 243-256.

CARrière, P. \& Monkewitz, P. A. 1999 Convective versus absolute instability in mixed RayleighBénard-Poiseuille convection. J. Fluid Mech. 384, 243-262.

Chapman, C. J. \& Proctor, M. R. E. 1980 Nonlinear Rayleigh-Bénard convection between poorly conducting boundaries. J. Fluid Mech. 101, 759-782.

Chомаz, J.-M. 2005 Global instabilities in spatially developing flows: non-normality and nonlinearity. Annu. Rev. Fluid Mech. 37 (1), 357-392.

Drazin, P. G. \& ReID, W. H. 2004 Hydrodynamic Stability. Cambridge University Press.

GRANDJEAN, E. 2008 Experimental investigation into localized instabilities of mixed RayleighBénard-Poiseuille convection. PhD thesis, Ecole Polytechnique Fédérale de Lausanne, Lausanne, Switzerland.

Hurle, D. T. J., Jakeman, E. \& Pike, E. R. 1967 On the solution of the Bénard problem with boundaries of finite conductivity. R. Soc. Lond. Proc. A 296, 469-475.

Kato, Y. \& Fujimura, K. 2000 Prediction of pattern selection due to an interaction between longitudinal rolls and transverse modes in a flow through a rectangular channel heated from below. Phys. Rev. E 62, 601-611.

Luijkx, J.-M., Platten, J. K. \& Legros, J. C. 1981 On the existence of thermoconvective rolls, transverse to a superimposed mean Poiseuille flow. Intl J. Heat Mass Transfer 24, 1287-1291.

Martinand, D., Carrière, P. \& Monkewitz, P. A. 2004 Envelope equations for the RayleighBénard-Poiseuille system. Part 2. Linear global modes in the case of two-dimensional nonuniform heating. J. Fluid Mech. 502, 175-197.

Martinand, D., Carrière, P. \& Monkewitz, P. A. 2006 Three-dimensional global instability modes associated with a localized hot spot in Rayleigh-Bénard-Poiseuille convection. J. Fluid Mech. 551, 275-301.

MÜLLER, H. W. 1990 Thermische Konvection in horizontaler Scherströmung. PhD thesis, Universitat des Saarlandes, Sarrebrück, Germany.

MÜLLER, H. W., LÜCKE, M. \& KAMPS, M. 1992 Transversal convection patterns in horizontal shear flow. Phys. Rev. A 45, 3714-3726.

NicOLAs, X. 2002 Revue bibliographique sur les écoulements de Poiseuille-Rayleigh-Bénard. Intl J. Therm. Sci. 41, 961-1016.

Nicolas, X., Luijkx, J. M. \& Platten, J. K. 2000 Linear stability of mixed convection flows in horizontal rectangular channels of finite transversal extension heated from below. Intl J. Heat Mass Transfer 43, 589-610.

Oppenheim, A. V. \& Schafer, R. W. 1989 Discrete-Time Signal Processing. Prentice-Hall.

Ouazzani, M. T., Caltagirone, J. P., Meyer, G. \& Mojtabi, A. 1989 Etude numérique et expérimentale de la convetion mixte entre deux plans horizontaux á température différentes. Intl J. Heat Mass Transfer 32, 261-269.

Ouazzani, M. T., Platten, J. K., Müller, H. W. \& LüCKe, M. 1995 Etude de la convection mixte entre deux plans horizontaux à températures différentes - III. Intl J. Heat Mass Transfer 38, $875-886$.

Pier, B. \& Huerre, P. 2001 Nonlinear synchronization in open flows. J. Fluids Struct. 15 (3-4), 471-480. 
Pier, B., Huerre, P. \& Chomaz, J.-M. 2001 Bifurcation to fully nonlinear synchronized structures in slowly varying media. Physica D 148, 49-96.

Sameen, A. \& Govindarajan, R. 2007 The effect of wall heating on instability of channel flow. $J$. Fluid Mech. 577, 417-442.

SetTles, G. S. 2001 Schlieren and Shadowgraph Techniques. Springer.

SiLVESTON, P. L. 1958 Wärmedurchgang in waagerechten Flüssigkeitsschichten. Forsch. Ingenieurw. 24, 59-69.

Stengel, K. C., Oliver, D. S. \& Booker, J. R. 1982 Onset of convection in a variable-viscosity fluid. J. Fluid Mech. 120, 411-431.

SveEN, J. K. 2004 An introduction to MatPIV v.1.6.1. Eprint no. 2, ISSN 0809-4403. Department of Mathematics, University of Oslo, Oslo, Norway. http://www.math.uio.no/ jks/matpiv.

TRAINOFF, S. P. 1997 Rayleigh-Bénard convection in the presence of a weak lateral flow. PhD thesis, University of California, Santa Barbara, CA.

White, F. M. 1974 Viscous Fluid Flow. McGraw-Hill. 